\title{
Lipmann-Schwinger Equations for a System of Multiband Energy-Eigenvalue Spectrum
}

\author{
M.A. Grado-Caffaro and M. Grado-Caffaro \\ M.A. Grado-Caffaro and M. Grado-Caffaro- Scientific Consultants, C/ Julio Palacios 11, 9-B, 28029-Madrid \\ (Spain); www.sapienzastudies.com; \\ Email: ma.grado-caffaro@sapienzastudies.com
}

\begin{abstract}
For the first time, we establish the Lippmann-Schwinger equations for a system of multiband energy-eigenvalue spectrum so that the involved time-independent Schrödinger wavefunctions become matrix elements. As a matter of fact, a supremum-seminorm of the total wavefunction matrix is estimated in terms of the same seminorm for the partial wavefunction matrix and for two key matrices of operators. In addition, an associated matrix-tensor formalism is presented.
\end{abstract}

Keywords: Lippmann-Schwinger equations; multiband eigenvalue spectrum; supremum-seminorm; matrices of operators; matrix formalism.

\section{Introduction}

Treating by using elegant mathematical-physics methods, quantum systems of multiband energyeigenvalue spectra is certainly interesting because these systems play an important role in several areas of Physics. Let us regard, for instance, quantum systems of two-band energy eigenvalue spectra [1,2]. A typical example of these systems can be found in magnetism of amorphous solids [1-3]. In particular, we refer to determine the magnetic susceptibility of amorphous solids [1-3]. In this context, a Wannier-type representation was employed in ref.[1] while a generalization of this representation, by introducing a matrix formalism, was done in ref.[2]. Really, the physics of condensed matter exhibits a number of examples of quantum systems with multiband eigenvalue spectra. Consider, for instance, a significant two-band eigenvalue spectrum consisting of spin-up and spin-down electronic bands (as, for example, in metamagnetic systems $[4,5]$ and nanophysics [6-10]) and a typical system with three-band spectrum as, for instance, a semiconductor with the conduction band, the valence band, and the forbidden band (band gap). Certainly, systems with two or three eigenvalue bands are relatively frequent in Physics. Therefore, it is natural to think on generalizing for many bands so that elaborating analytical models to characterize multiband systems is desirable.

On the other hand, the so-called Lippmann-Schwinger model is suitable to discuss relevant issues in Nuclear Physics as, for example, neutron-nucleus scattering [11] and in Solid State Physics as, for instance, problems related to optical potential [12]. It is well-known (see, for instance, ref.[11]) that the Lippmann-Schwinger approach consists of a system of equations (one equation for each quantum state) relative to the time-independent non-relativistic or relativistic Schrödinger equation with a potential viewed as a perturbation. Here, we will consider the static non-relativistic Schrödinger equation with a time-independent external potential. Within this picture, electron-atom scattering or electron-molecule scattering may be considered by employing the Lippmann-Schwinger equations in relation to a multiband energy-eigenvalue spectrum. In fact, among other things, we will establish a matrix formalism to describe the aforementioned scattering.

\section{Theory}

We consider the $\psi^{(+)}$Lippmann-Schwinger equations, which read (see, for example, refs.[11,12]):

$$
\left|\psi_{n}\right\rangle=\left|\varphi_{n}\right\rangle+\left(E_{n}+i \varepsilon-\hat{H}_{0}\right)^{-1} \hat{V}\left|\psi_{n}\right\rangle
$$


where $\left|\psi_{n}\right\rangle$ denotes elastic (coherent) total wavefunction $(n=1,2, \ldots),\left|\varphi_{n}\right\rangle$ is partial wavefunction, $\hat{H}_{0}$ indicates field-free Hamiltonian operator, $E_{n}$ is the $n^{\text {th }}$ energy eigenvalue, $\hat{V}$ is the involved, timeindependent, potential-energy operator, and $i$ is the imaginary unit. We have the time-independent Schrödinger equations, namely, $\hat{H}_{0}\left|\varphi_{n}\right\rangle=E_{n}\left|\varphi_{n}\right\rangle$ and $\left(\hat{H}_{0}+\hat{V}\right)\left|\psi_{n}\right\rangle=E_{n}\left|\psi_{n}\right\rangle$.

Now we are interested in extrapolate formula (1) to a quantum system of multiband energyeigenvalue spectrum. In this case, the corresponding time-independent Schrödinger equations are:

$$
\begin{gathered}
\hat{H}_{0}\left|\varphi_{m n}\right\rangle=E_{m n}\left|\varphi_{m n}\right\rangle \\
\left(\hat{H}_{0}+\hat{V}\right)\left|\psi_{m n}\right\rangle=E_{m n}\left|\psi_{m n}\right\rangle
\end{gathered}
$$

where $m$ is the band index and $n$ labels the (bound) states in each band; we have that $m=1,2, \ldots, N$ (where $N$ is the number of bands) and $n=1,2, \ldots$ We may also consider the total Hamiltonian operator namely $\hat{H} \equiv \hat{H}_{0}+\hat{V}$ such that $\hat{H}\left|\psi_{m n}\right\rangle=E_{m n}\left|\psi_{m n}\right\rangle$ (see eq.(3)). On the other hand, let us assume that the ground state of the system is a state such that all the states in the $m=1$ band are occupied.

Therefore, the Lippmann-Schwinger equations for the system of multiband spectrum in question are:

$$
\left|\psi_{m n}\right\rangle=\left|\varphi_{m n}\right\rangle+\left(E_{m n}+i \varepsilon-\hat{H}_{0}\right)^{-1} \hat{V}\left|\psi_{m n}\right\rangle
$$

which implies:

$$
\sum_{m, n}\left|\psi_{m n}(\vec{r})\right\rangle=\sum_{m, n}\left|\varphi_{m n}(\vec{r})\right\rangle+\sum_{m, n}\left(E_{m n}+i \varepsilon-\hat{H}_{0}\right)^{-1} \hat{V}(\vec{r})\left|\psi_{m n}(\vec{r})\right\rangle
$$

Note that in eq.(5) it is indicated the explicit dependence on the spatial coordinates of the involved wavefunctions (which, of course, are complex-valued functions) and the external potential. Also in relation to eq.(5), for the sake of brevity of notation, we omit the range of values of $m$ and $n$ into the summation symbols.

It is clear that, in both formulae (4) and (5), the total wavefunction and the partial one are matrix elements so that we may speak of the matrices, namely, $\Psi \equiv\left(\psi_{m n}\right)$ and $\Phi \equiv\left(\varphi_{m n}\right)$, where, for the sake of brevity of notation, dependence on spatial coordinates and Dirac's notation of "ket" vectors is not shown explicitly. In order to elaborate later a matrix-tensor formalism, we are interested in considering square matrices so that we define $\psi_{m n}=\varphi_{m n}=0$ for $m=N+1, N+2, \ldots$

Now, instead of utilizing the usual norm of the wavefunction Hilbert space, this norm being unity (normalized wavefunctions), then we define the following supremum-seminorms as figures of merit of the quantum multiband states in question:

$$
\begin{array}{r}
\left.\|\Psi\| \equiv \sup _{\vec{r} \in R}\left|\sum_{m, n}\right| \psi_{m n}(\vec{r})\right\rangle \mid \\
\left.\|\Phi\| \equiv \sup _{\vec{r} \in R}\left|\sum_{m, n}\right| \varphi_{m n}(\vec{r})\right\rangle \mid
\end{array}
$$

where $R$ is the involved spatial region which is assumed to be compact. Note that the existence of the right-hand side of (6) and (7) is manifest because the functions $\psi_{m n}(\vec{r})$ and $\varphi_{m n}(\vec{r})$ are twofold differentiable on a compact set so they are bounded in this set so the sum indicated in (6) and (7) is also bounded in $R$.

Notice that, in effect, expressions (6) and (7) define seminorms since, although the direct assertion of the first axiom of a norm is fulfilled (if $\Psi \equiv 0$ or $\Phi \equiv 0$, the right-hand side of (6) or (7) is zero), then the reciprocal assertion is not fulfilled, that is, if the right-hand side of (6) or (7) is zero, then the lefthand side can be different from zero.

Taking supremum at both sides of (5) and using relations (6) and (7), it follows:

$$
\|\Psi\| \leq\|\Phi\|+\|\hat{\Gamma}\|\|\hat{V}\|\|\Psi\|
$$


where $\hat{\Gamma}$ is a matrix whose elements are linear operators such that $\hat{\Gamma} \equiv\left(\hat{\gamma}_{m n}\right)$ and $\hat{\gamma}_{m n} \equiv\left(E_{m n}+i \varepsilon-\hat{H}_{0}\right)^{-1}$ which are bounded. Observe that $\|\hat{\Gamma}\|$ and $\|\hat{V}\|$ are seminorms, which can be viewed as "strengths" of the respective operators $\hat{\Gamma}$ and $\hat{V}$. One has:

$$
\begin{gathered}
\|\hat{\Gamma}\| \equiv \sup _{\Psi \neq 0} \frac{\|\hat{\Gamma} \Psi\|}{\|\Psi\|} \\
\|\hat{V}\| \equiv \sup _{\Psi \neq 0} \frac{\|\hat{V} \Psi\|}{\|\Psi\|}
\end{gathered}
$$

Similarly, we may regard $\|\Psi\|$ and $\|\Phi\|$ as "strengths" of $\Psi$ and $\Phi$, respectively. On the other hand, if we adopt the following norms rather than the seminorms (6) and (7):

$$
\begin{array}{r}
\left.\|\Psi\| \equiv \sup _{\vec{r} \in R} \sum_{m, n} \| \psi_{m n}(\vec{r})\right\rangle \mid \\
\left.\|\Phi\| \equiv \sup _{\vec{r} \in R} \sum_{m, n} \| \varphi_{m n}(\vec{r})\right\rangle \mid
\end{array}
$$

as well as for the operators $\hat{\Gamma}$ and $\hat{V}$, then it is easy to see that inequality (8) holds where, of course, now the symbol $\|\cdot\|$ means norm.

On the other hand, relationship (5) can be expressed as follows:

$$
\sum_{m, n} \psi_{m n}=\sum_{m, n} \varphi_{m n}+\operatorname{tr}((\hat{\Gamma} \hat{V}) \otimes \Psi)
$$

where $\operatorname{tr}$ denotes trace, the tensorial product is not the standard tensor product in the Krönecker sense used in quantum mechanics but the dyadic-tensor product of matrices $[2,13]$. This product comes from multiplying dyadically two vectors $[2,13]$. As in preceding notation, for the sake of brevity, in relation (13) we abandon Dirac's notation and dependence on spatial coordinates is not shown explicitly.

Taking supremum at both sides of (13) and employing formulas (6) and (7), one gets:

$$
\|\Psi\| \leq\|\Phi\|+\sup _{\vec{r} \in R}|\operatorname{tr}((\hat{\Gamma} \hat{V}) \otimes \Psi)|
$$

By combining inequalities (8) and (14), we find:

$$
\sup _{\vec{r} \in R}|\operatorname{tr}((\hat{\Gamma} \hat{V}) \otimes \Psi)|=c\|\hat{\Gamma}\|\|\hat{V}\|\|\Psi\|
$$

where $c$ is a number such that $0<c \leq 1$ or $c>1$.

Then we enunciate:

Theorem 1.- There exists a real number namely $c(0<c \leq 1$ or $c>1)$ such that equality (15) is satisfied.

On the other hand, from formula $(8)$ it follows:

$$
\frac{\|\Phi\|}{\|\Psi\|} \geq 1-\|\hat{\Gamma}\|\|\hat{V}\|
$$

Expression (16) gives a lower bound for the ratio of the "strength" of the partial wavefunction matrix to the "strength" of the total wavefunction matrix. The above bound depends significantly on the seminorms of $\hat{\Gamma}$ and $\hat{V}$.

Now we have:

Definition 1.- The so-called transition operator namely $\hat{T}$ is such that $\hat{T}\left|\varphi_{m n}\right\rangle=\hat{V}\left|\psi_{m n}\right\rangle$.

Definition 2.- We define the scattering operator namely $\hat{S}$ such that $\hat{S}\left|\varphi_{m n}\right\rangle=\left|\psi_{m n}\right\rangle$.

In addition, one enunciates:

Proposition 1.- It is verified:

$$
\|\Psi\| \leq\|\Phi\|(1+\|\hat{\Gamma}\|\|\hat{T}\|)
$$

where, of course, the seminorm of $\hat{T}$ is the same as for $\hat{\Gamma}$ and $\hat{V}$.

Proof: By using Definition 1, taking supremum at both sides of (5) and considering formulae (6) and (7), then relation (17) is easily found (let us regard expression (8)) 
Theorem 2.- There exists a real number namely $c$ such that $0<c \leq 1$ or $c>1$ and such that:

$$
\sup _{\vec{r} \in R}|\operatorname{tr}((\hat{\Gamma} \hat{T}) \otimes \Phi)|=c\|\hat{\Gamma}\|\|\hat{T}\|\|\Phi\|
$$

Proof: Taking supremum at both sides of (13), by using relations (6) and (7) as well as Definition 1 , then one gets:

(regard formula (14))

$$
\|\Psi\| \leq\|\Phi\|+\sup _{\vec{r} \in R}|\operatorname{tr}((\hat{\Gamma} \hat{T}) \otimes \Phi)|
$$

Combining now inequalities (17) and (19), one arrives at formula (18)

Proposition 2.- It is satisfied the following inequality:

$$
\|\hat{S}\| \leq 1+\|\hat{\Gamma}\|\|\hat{T}\|
$$

where, obviously, the seminorm of $\hat{S}$ is the same as for $\hat{\Gamma}, \hat{V}$ and $\hat{T}$.

Proof: Expression (20) comes straightforwardly from Definitions 1 and 2 combined with formulas (5), (6) and (7)

Corollary.- By Definition 2 and formulae (6) and (7), one gets $\|\Psi\| /\|\Phi\| \leq\|\hat{S}\|$ which, together with inequality $(20), \Rightarrow(17)$.

Finally, we consider the following tight-binding basis $[1,2]$ :

$$
\left|\psi_{m n}\right\rangle=\sum_{k}\left\langle\psi_{m k} \mid \psi_{m n}\right\rangle\left|\psi_{m k}\right\rangle
$$

where $k=1,2, \ldots$

Then one has:

Proposition 3.- It is verified that $[1,2]$ :

$$
\sum_{n}\left|\psi_{m n}\right\rangle\left\langle\psi_{m n}\left|\equiv \sum_{k}\right| \psi_{m k}\right\rangle\left\langle\psi_{m k}\right|
$$

Proof: By summing over the range $n=1,2, \ldots$ the "product" of the "ket" vectors on the left-hand side of (21) by the corresponding "bra" vectors and interchanging summation with respect to $n$ by summation relative to $k$, then the left-hand side of (22) becomes $\left\langle\psi_{m n} \mid \psi_{m n}\right\rangle^{2} \sum_{k}\left|\psi_{m k}\right\rangle\left\langle\psi_{m k}\right|$ with $\left\langle\psi_{m n} \mid \psi_{m n}\right\rangle=1$ so expression (22) is satisfied

Similarly to formula (21), we also consider the tight-binding basis:

$$
\left|\varphi_{m n}\right\rangle=\sum_{k}\left\langle\varphi_{m k} \mid \varphi_{m n}\right\rangle\left|\varphi_{m k}\right\rangle
$$

so that, as in relationship (22), we have:

$$
\sum_{n}\left|\varphi_{m n}\right\rangle\left\langle\varphi_{m n}\left|=\sum_{k}\right| \varphi_{m k}\right\rangle\left\langle\varphi_{m k}\right|
$$

Expressions (21) to (24) represent a useful formulation from the point of view of disordered systems in Condensed Matter Physics [1-3].

\section{Discussion}

It is well-known that problems upon operator theory concerning quantum perturbed systems [14-19] and related issues [20-25] have a great relevance in Mathematical Physics; our preceding formulation is an example of this. As a matter of fact, by using relations (6), (7), (9) and (10), we have developed an approach whose main results are relationships (15) and (18). In our formulation, it has been employed (see relations (6) and (7)) a seminorm that can be regarded as unusual given that, of course, the usual norm on the Hilbert space of the wavefunctions considered here is $\left\langle\psi_{m n} \mid \psi_{m n}\right\rangle^{1 / 2}=1$. However, the aforementioned seminorm and the norm defined in formulae (9) and (10) which, of course, correspond to non-Hilbert space but to semi-Banach or Banach space, then constitute, say, key signatures or figures of merit ("strengths") to characterize the matrices $\Psi$ and $\Phi$. We may speak of a semi-Banach space as a complete seminormed real or complex vector space. On the other hand, from inequality (16) one gets the 
relative deviation $(\|\Psi\|-\|\Phi\|) /\|\Psi\| \leq\|\hat{\Gamma}\|\|\hat{V}\|$ so that an upper bound of this relative deviation equals the product of the "strengths" of $\hat{\Gamma}$ and $\hat{V}$.

\section{Concluding Remarks}

We have shown that, by utilizing the four linear operators $\hat{\Gamma}, \hat{V}, \hat{T}$ and $\hat{S}$, an elegant mathematical representation has been achieved within an unusual framework because the usual Hilbert-space structure has not been considered but the Banach (or, more precisely, semi-Banach) space structure. This fact opens new avenues to treat a number of relevant issues on Mathematical Physics in an alternative way which could be very attractive for future studies.

\section{References}

1. R.M. White in "Tetrahedrally bonded amorphous semiconductors" (M.H. Brodsky, S. Kirkpatrick, and D. Weaire, Eds.), AIP Conf. Proc. No.20 (1974) 44-46.

2. M.A. Grado-Caffaro, M. Grado-Caffaro, Mod. Phys. Lett. B 18 (2004) 1449-1452.

3. M.A. Grado-Caffaro, M. Grado-Caffaro, Act. Pass. Electronic Comp. 17 (1994) 253-255.

4. N.H. Duc, D. Givord, C. Lacroix, C. Pinettes, Europhys. Lett. 20 (1992) 47-52.

5. M.A. Grado-Caffaro, M. Grado-Caffaro, Act. Pass. Electronic Comp. 24 (2001) 63-67.

6. M.A. Grado-Caffaro, M. Grado-Caffaro, Superlat. Microstruct. 48 (2010) 443-449.

7. A. Soldatov, N. Bogolubov (Jr.), S. Kruchinin, Condens. Matter Phys. 9 (2006) 151-159.

8. V. Ermakov, S. Kruchinin, H. Hori, A. Fujiwara, Int. J. Mod. Phys. B 21 (2007) 1827-1835.

9. S. Kruchinin, H. Nagao, S. Aono, Int. J. Mod. Phys. B 26 (2012) 1230013-1230067.

10. M. Apostol, Phys. Lett. A 372 (2008) 5093-5095.

11. J.J. Sakurai, J.J. Napolitano: Modern Quantum Mechanics (Addison-Wesley, second edition, 2010).

12. M.A. Grado-Caffaro, M. Grado-Caffaro, Mod. Phys. Lett. B 26 (2012) 12502101-12502105.

13. M.A. Grado-Caffaro, M. Grado-Caffaro, Adv. Stud. Theor. Phys. 6 (2012) 1183-1188.

14. T. Kato: Perturbation theory for linear operators (Springer-Verlag, 1966).

15. L. Parnovski, A.V. Sobolev, Ann. H. Poincaré 2 (2001) 573-581.

16. A.V. Sobolev, Ann. H. Poincaré 6 (2005) 31-84.

17. J. Feldman, H. Knörrer, E. Trubowitz, Invent. Math. 100 (1990) 259-300.

18. J. Feldman, H. Knörrer, E. Trubowitz, Comment. Math. Helvetici 66 (1991) 557-579.

19. Yu. Karpeshina: Perturbation theory for the Schrödinger operator with a periodic potential. Lecture Notes in Mathematics, vol. 1663 (Springer-Verlag, Berlin, 1997).

20. A. Mohamed, J. Math. Phys. 38 (1997) 4023-4051.

21. E. Korotyaev, A. Pushnitski, Bull. London Math. Soc. 35 (2003) 770-776.

22. B.E.J. Dahlberg, E. Trubowitz, Comment. Math. Helvetici 57 (1982) 130-134.

23. M. Skriganov, Invent. Math. 80 (1985) 107-121.

24. D. Shenk, M. Shubin, Math. USSR Sbornik 56 (1987) 473-490.

25. F. Schwabl: Advanced Quantum Mechanics (Springer-Verlag, Heidelberg, 2004). 